\title{
Franz Grillparzer - der österreichische Klassiker?
}

\author{
Walter Weiss
}

\section{Vorbemerkung}

Der Anlaß für meinen Vortrag ist Grillparzers 200. Geburtstag. Ich wähle als Einstieg ein früheres Geburtstagsgedenken.

\section{Einstieg}

Zum 80. Geburtstag des noch Lebenden identifizierte der österreichische Publizist Ferdinand Kürnberger den Jubilar mit Österreich und lieferte damit nicht zuletzt auch einen Beitrag zum heutigen Thema und Anlaß. ${ }^{1}$ Kürnbergers besondere Perspektive besteht darin, daß er Grillparzers Leben und Schaffen mit dem Verhalten und dem Zustand Österreichs von 1791 bis 1871 synchronisiert und in eine Opposition zu Deutschland bringt.

Dieses Deutschland charakterisiert er als dynamisch, fortschrittlich, zukunftsorientiert - unmittelbar vor der vor hundertzwanzig Jahren erfolgten Proklamation des preußisch-deutschen Kaiserreiches. Österreich hebt er davon ab als geschockt und paralysiert durch die Französische Revolution, durch die Hinrichtung der Tochter Maria Theresias, als sich abwendend und zurückweichend von den Reformen Josephs II., als schwächlich und regressiv. Der Kernsatz dieses Geburtstagsgedenkens:

Grillparzer war in jedem Sinne berufen, ein großer deutscher Dichter zu werden. Er wurde nur Osterreichs Grillparzer. (284)

Damit erklärt Kürnberger auf seine Weise - im kritischen Sinn - Grillparzer zum österreichischen Klassiker. Klassiker verstanden als repräsentativer, identitätsstiftender Nationalautor, wie ihn bereits Goethe in seiner Schrift über den "Literarischen Sanscoulottismus" 2 bestimmte.

Auch wenn man einschränkend Kürnbergers Position als die eines deutschnational gewordenen österreichischen Liberalen berücksichtigt, bleibt diese seine Positionbestimmung bedenkenswert, auch und gerade heute. Drängen sich doch in

1. Ferdinand Kürnberger, "Oesterreich's Grillparzer. 14.1.1871." In: Literarische Herzenssachen. Wien 1877, S. 281-298.

2. Goethes Werke. Hamburger Ausgabe. 4. Aufl. Bd. 12. Hamburg 1960, S. 239244. 
der Situation 1991 Aktualisierungen der Situation von 1871 auf, angesichts eines in seiner Identität etwas verunsicherten Österreich (Stichwort: Neutralitätsdiskussion, EG-Diskussion), gegenüber einem vereinten Deutschland, mit seiner Wirtschaftsmacht, die auch umgesetzt wird in eine machtbewußte Außenkulturpolitik, mit seiner führenden Rolle in der EG, vor deren noch verschlossenen Türen Österreich als ein bis auf weiteres zum Anstellen aufgeforderter Bittsteller steht.

\section{Annäherung an die, Abhebung von der deutschen Klassik}

Das Thema "österreichischer Klassiker" ist bei Grillparzer noch in einem anderen Sinn mit Bezug auf Deutschland bedeutungsvoll geworden, - auf ein anderes Deutschland allerdings als das von 1871 -: in seinem Verhältnis zur deutschen Klassik.

Grillparzer selbst hat es ins Spiel gebracht, wie mit dem folgenden Epigramm, das in der Opposition zwischen "vorwärts" und "stehen bleiben" an Kürnbergers Perspektive erinnert:

Nur weiter geht das tolle Treiben,

Von vorwärts! vorwärts! erschallt das Land;

Ich möchte, wär's möglich, stehenbleiben,

Wo Schiller und Goethe stand. ${ }^{3}$

Wendet sich damit Grillparzer gegen den neudeutschen Fortschritt, (vor dem März 1848) so sah der Herausgeber der historisch-kritischen Ausgabe seiner Sämtlichen Werke, August Sauer, mit Grillparzer die "jahrhundertelange" österreichische "Sonderentwicklung", die "dem vorwärtsstrebenden Zuge deutschen Geistesleben völlig entfremdet war", den Anschluß an die deutsche Klassik im Zeichen der deutschen Kulturnation herstellen:

ein neuer Klassiker" österreichischer Prägung habe "den eisernen Bestand des literarischen Nationalve $m$ ögens [...] vermehrt. ${ }^{4}$

Und Sauers Fortsetzer, Reinhold Backmann, hatte 1938 die Vision eines würdigen Denkmals für den "künftigen und endgültigen Grillparzer", situiert auf

3. Franz Grillparzer, Sämtliche Werke. Ausgewählte Briefe, Gespräche, Berichte. Bd. 1, 2. Hrsg. von Peter Frank u. Karl Pömbacher. München 1969, S. 1257. Mehrmals, in leicht veränderter Fassung, 1844, 1846, 1847, 1851, 1852, 1853, $1854,1858$.

4. Grillparzers sämtliche Werke. Fünfte Ausgabe in zwanzig Bänden. Vorbericht und Einleitungen. Bd. 1/2, Stuttgart: o.J., S. $11 \mathrm{f}$. 
dem "Kahlenberg", zugleich "Walhalla eines Einzigen" und "Tempel [...] gleich dem auf Sestos" aus Grillparzers Hero-Drama ${ }^{5}$ - in Österreich angesiedelter Klassizismus also, mit antiken und deutsch-nordischen (wagnerianischen) Zügen und ein Hinweis auf die bayrische Walhalla. Gegenläufig zu dieser als Aufwertung gemeinten Annäherung wird Grillparzer von der deutschen Klassik als Epigone abgerückt und abgewertet. Dies geschah etwa bei Wilhelm Scherer, zeitlich zusammenfallend mit seiner Entscheidung gegen sein Herkunftsland Österreich und für das neugegründete preußisch-deutsche Reich $(1872)^{6}$, ästhetisch besonders nachdrücklich jedoch bei dem Goethe-Monographen Friedrich Gundolf $(1931)^{7}$ und, nicht ohne Rückbezug auf ihn, bei Gerhart Baumann (1951, ${ }_{1966)}^{8}$; bei ihm allerdings insofern ambivalent, als er nicht dabei stehenbleibt, die gebrochene Sprache Grillparzers mit der ungebrochenen eines Goethe oder eines Kleist zu kontrastieren, sondern darin auch in die Zukunft, zur Moderne hinführende Züge entdeckt.

Damit schließt er aber auf seine Weise an die "Identifikation Grillparzers mit einem 'größeren Österreich'" an, der ich mich jetzt zuwende. Sie ging darauf aus, das einschränkende "nur" in Kürnbergers "nur Österreichs Grillparzer" zu tilgen und die von Kürnberger getadelte Einschränkung zu einer Auszeichnung umzufunktionieren.

\section{Identifikation mit dem 'größeren Österreich'}

Es ist wohl mehr als ein Zufall, daß Hugo von Hofmannstahl angesichts des drohenden Unterganges der Habsburgermonarchie im Ersten Weltkrieg Grillparzer als Gegenhalt, als Inbegriff des "reinen österreichischen Selbst" beschwor, in dem sich Vergangenheit und Gegenwart verbindet ("Grillparzers politisches Vermächtnis", 1915) ${ }^{9}$ : "Grillparzer geht aus dem alten Österreich hervor und ragt in das neue hinein; er steht mitten zwischen der Zeit Maria Theresias und

5. Reinhold Backmann, Der Kampf um das endgültige Grillparzer-Bild beginnt (=Schriften des Pädagogischen Instituts der Stadt Wien 16), Wien-Leipzig o.J. (1938), S. 13.

6. Wilhelm Scherer, "Franz Grillparzer. Beiträge zu seinem Verständnis." In: Vorträge und Aufsätze zur Geschichte des geistigen Lebens in Deutschland und Österreich. Berlin 1874.

7. Friedrich Gundolf, "Grillparzer." In: Jahrbuch des Freien Deutschen Hochstifts 1931, S. 9-94.

8. Gerhart Baumann, Grillparzer. Sein Werk und das österreichische Wesen. Freiburg 1954; Franz Grillparzer. Dichtung und österreichische Geistesfassung. Frankfurt/M. 1966.

9. Hugo von Hofmannstahl, Ausgewählte Werke in zwei Bänden. Hrsg. von Rudolf Hirsch. Bd. 2, Frankfurt/M. 1961, S. 582-587. 
unserer eigenen. Sein Charakter, der hier und dorthin paßt [...] gibt uns den Begriff eines unzerstörbaren österreichischen Wesens".

Später verlegte Hofmannsthal Grillparzers historische Herkünfte noch weiter zurück, wenn er ihm bescheinigt, mit dem Bruderzwist die vollgültige Darstellung "einer vergangenen, im wesentlichen aber noch fortwirkenden Epoche [der des 17. Jhs. W.W.] ganz zu geben", was nach seiner Ansicht "nur einem Österreicher vielleicht" "gelingen" "konnte". ${ }^{10}$ Dies fügt sich zum Anschluß Grillparzers an die Traditionen einer "barocken Literatur- und Theaterlandschaft", wie ihn (damals) Josef Nadler, Otto Rommel u.a. vollzogen.

Es ist bezeichnend für das auf diese Weise aufgeladene österreichische Selbstbewußtsein, wie anders nun Josef Nadler die Begegnung Grillparzers mit Goethe 1826 darstellte als vor ihm Wilhelm Scherer. Während Scherer den "knabenhaft" in Tränen ausbrechenden Grillparzer einem Goethe gegenüberstellte, der ihn schonend väterlich behandelt (im AnschluB an Grillparzers Selbstbiographie und seine Tagebuchaufzeichnungen), faßt Nadler die Begegnung ideologisch-stilisierend so zusammen: "Goethe und Grillparzer: Begegnung und Vorübergleiten zweier Kulturräume und zweier Zeitalter, ihres eigengesetzlichen Daseins und ihrer Vertreter." Dabei erscheint Grillparzer der deutschen Klassik gegenüber als Vertreter des "älteren österreichischen Vorgangs." 11

Dieser "ältere Vorgang", den Hofmannsthal noch unentschieden zwischen Maria Theresia und dem siebzehnten Jahrhundert angesetzt hat, wurde nun von Nadler u.a. geistes- und theatergeschichtlich mehr und mehr mit den Barocktraditionen gleichgesetzt; bis hin zu dem Punkt, wo man Grillparzers barockes "Urerlebnis" gegen sein angeblich bloß aufgesetztes "Bildungserlebnis" der Aufklärung , des Josephinismus, gewertet als unösterreichischen Fremdkörper, ausspielte (E. Alker). ${ }^{12}$

Gegen diese "Unvereinbarkeitsthese" stellte Roger Bauer seine These von der Kontinuität zwischen österreichischem Barock und österreichischer Aufklärung: Josephinismus als "ernüchtertes Barock." ${ }^{13}$ Damit ließ sich das angeblich Unvereinbare wieder miteinander verbinden.

10. Hugo von Hofmannsthal, Rede auf Grillparzer (1922). Ebd. S. 651-671, zitierte Stelle, S. 664.

11 Josef Nadler, "Goethe und Grillparzer." In: Corona 3 (1933), S. 511; Literaturgeschichte der deutschen Stämme und Landschaften, Bd. 4, Regensburg 1928, S. 434f.

12. Ernst Alker, Franz Grillparzer. Ein Kampf um Leben und Kunst. Marburg a. L. 1930, S. $96 f$.

13. Roger Bauer, "Konstanten der österreichischen Literatur vom Barockzeitalter bis zum Spät- und Nachjosephinismus." In: Die Welt als Reich Gottes. Wien 1974, S. $13-45$. 
Allerdings bleibt Zdenko $\$ krebs 1976 in seinem Grillparzerbuch nachdrücklich vorgetragene Warnung vor der Umwandlung des "Epochenbegriffs Barock" "in einen geschichtstranszendenten integrierenden Bestandteil des 'Österreichischen"14 sehr wohl zu beachten.

Übereinstimmend damit wurden die Stimmen immer lauter, die eine "unbefangene Bestimmung von Grillparzers historischem Ort" (F. Sengle $1980)^{15}$ fordern und damit sowohl meinen, daß man "mit seiner Zeitgenossenschaft wirklich ernst" machen, wie auch, daß man seine Orientierung an Traditionen des achtzehnten Jahrhunderts stärker beachten solle, - "wodurch (dann) das Wort vom 'österreichischen Klassiker' einen festeren Inhalt" gewinne.

Das richtet sich nicht nur gegen die Versuche, Grillparzer "einseitig in der Richtung auf deutsche Klassik" oder "katholischen Barock" zu stilisieren, sondem zugleich auch gegen seine "Modernisierung" in Richtung auf den "Realismus" des neunzehnten Jahrhunderts oder auf den "Wiener Impressionismus".

Diese "Modernisierung" hat vor allen Gerhart Baumann betrieben, der in der zweiten Fassung seines Grillparzerbuches 1966 verallgemeinernd behauptet: "Das Komplexe, Vielverschlungene, Verschlossene der Grillparzerschen Dichtung und Geistigkeit [...] entziffert sich eigentlich erst ganz im nachfolgenden österreichischen Schrifttum des neunzehnten und zwanzigsten Jahrhunderts" ${ }^{16}$, etwa im Werk Hofmannsthals und Schnitzlers.

Die damit verbundene These, daß "diese ungebrochne Art des Rückschließens [...] innerhalb des deutschen Sprachraums allein in Österreich möglich und erlaubt" sei, bringt den hier verfolgten Prozeß der fortschreitenden Identifizierung Grillparzers mit dem "größeren Österreich" zu einem kaum mehr zu überschreitenden Abschluß.

\section{Kritik am "größeren Österreich"}

Kritik daran ließ nicht auf sich warten. Claudio Magris diagnostizierte, ambivalent zwischen Entlarvung und Verzauberung, das "größere Österreich" als "habsburgischen Mythos" (it. 1963, dt. 1966, 1988) - als Produkt eines ideologisch-ästhetischen Prozesses der Verdrängung und stilisierenden Transformation der historischen Wirklichkeit, dem er allerdings später auch zukunftsweisende Qualität als utopischer Vorschein "Mitteleuropas" zuschrieb.

Und wiederum wurde Grillparzer dabei zur Schlüsselgestalt: "In seinem Werk,

14. Zdenko Skreb, Grillparzer. Kronberg/Ts. 1976, S. 229.

15. Friedrich Sengle, Biedermeierzeit. Bd. 3: Die Dichter. Stuttgart 1980, S. 131, $58,132$.

16. Gerhart Baumann, Franz Grillparzer. Dichtung und österreichische Geistesverfassung. Frankfurt/M., Bonn 1966. S. 12f. 
seiner Empfindungswelt und seiner Schöpfung spiegelt sich ganz Österreich samt seiner widerspruchsvollen, bis zum Zusammenbruch des Jahres 1918 ungelösten Problematik. Seine Dichtung ist der erste, vollendete Ausdruck des habsburgischen Mythos."

Grillparzers gesamtes Werk durchziehen ein paar endlos abgewandelte, doch im wesentlichen einheitlich getönte Mythosworte und Grundthemen [...] Selbstbewahrung, Entsagung, Treue, Beharren, Besitzen und Verlieren, Entpersönlichung, Selbstentfremdung. Es ist dies ein typischer, klar aus dem Barock herrührender Komplex. ${ }^{17}$

So Claudio Magris. Seine Problematisierung des "größeren Österreich" brachte also weniger einen Paradigmenwechsel als Vorzeichenveränderungen und ließ die Stellung Grillparzers als "klassischer österreichischer Dichter" (97) unangetastet. Auch bei Magris, der die Geburtsstunde des "habsburgischen Mythos" mit dem hinhaltenden Widerstand Österreichs gegen die französische Revolution und ihre nationalistischen und demokratischen Folgen koppelt, fehlt, wie wir bemerkt haben, die Rückführung Grillparzers auf Barocktraditionen nicht.

Ähnlichen österreichischen Kontinuitäten, als Grundlage für die Grillparzerdeutung, begegnen wir dort, wo Historiker (wie Helmut Rumpler) ${ }^{18}$ prägende Ordnungsvorstellungen, wie das z.T. bis heute weiterlebende hierarchische Obrigkeitsmodell auf das Bündnis zwischen katholischer Gegenreformation und der Durchsetzung des Absolutismus zurückführen. Auch in dem von Leslie Bodi herausgearbeiteten Widerspruch zwischen (katholischer) Aufklärung und Absolutismus, ${ }^{19}$ in der Durchsetzung der Aufklärung mit absolutistischen Mitteln unter Joseph II., kann man diese österreichische Kontinuität wiederfinden. Das Ordnungsdenken österreichischer Josephinisten, nicht zuletzt auch eines Franz Grillparzer, läßt sich damit verbinden. Man denke etwa nur an sein vieldiskutiertes Radetzky-Gedicht. ${ }^{20}$

17. Claudio Magris, Der habsburgische Mythos in der österreichischen Literatur. 2. Aufl. Salzburg 1988, S. 97-99.

18. Vgl. dazu Walter Weiss, "Zur Diskussion über die österreichische Literatur." In: Schweiz-Österreich. Ähnlichkeiten und Kontraste. Graz-Wien 1986, S. 69-82.

19. Leslie Bodi, Tauwetter in Wien. Zur Prosa der österreichischen Aufklärung 17811795. Frankfurt/M. 1977.

20. Franz Grillparzer, Sämtliche Werke. Ausgewählte Briefe, Gespräche, Berichte. Bd. 1, 2. Aufl., München 1969, S. 318 ("Feldmarschall Radetzky"): "In deinem Lager ist Österreich / Wir andern sind einzelne Trümmer." Vgl. aber auch Grillparzers Emüchterung, Bd. 4, S. 716 (Tgb. 4024, 2. Hälfte Sept. 1849). 


\section{Widersprüche, Öffnungen}

Soeben ist das Stichwort "Widerspruch" gefallen. Es eignet sich gut zur Charakterisierung neuer und neuester Beiträge, die gemeinsam haben, daß sie sich gegen die seinerzeit von Backmann ausgegebenen Parole Der Kampf um das endgültige Grillparzer-Bild beginnt (1938) richten.

Bezeichnend dafür ist etwa, daß Gerhard Scheit seine "rowohlt-monographie" von 1989 mit einer Berufung auf Ferdinand Kürnbergers Nachruf vom 23.1.1872 eröffnet, ${ }^{21}$ den dieser, wie folgt, beschloß: "Zur Psychologie Österreichs ist die Biographie Grillparzers unentbehrlich. Man wird diese Biographie jedenfalls schreiben, aber verdorren soll die Hand, die nicht ihre ganze Wahrheit schreiben wird!"22 Gemeint ist damit nicht eine harmonisierende Abrundung, sondern Offenheit für den Widerspruch zwischen dem "weichen, passiven Österreicher" und seinen rebellischen "starken Leidenschaften." Diesen Widerspruch sah bereits Kürnberger mit den Widersprüchen der Zeit Grillparzers zusammen: Dafür gewinnt nun die Grillparzer-Forschung ein empfänglicheres Organ. ${ }^{23}$

Auf der sprachlich-äthetischen Ebene geschah das, noch weniger beabsichtigt als im nachhinein, durch das Aufeinandertreffen von widerstreitenden Thesen und Forschern. Da waren auf der einen Seite die, welche Grillparzer die "eigene Gestalt, die selbständige Sprache" absprachen (Baumann), ${ }^{24}$ "keiner" habe "so matt und zag" gesprochen, "schwach, flau, klapprig" sei "fast überall sei Ton" (Gundolf). ${ }^{25}$ Auf der anderen Seite standen die, welche den Einbruch der modernen Psychologisierung, der Spannungen zwischen dem Bewußten und dem Unter- und Unbewußten in der klassischen Mustern noch verpflichteten Dramensprache Grillparzers erkennen (Joachim Kaiser). ${ }^{26}$ Wieder andere sahen darin ältere, spezifisch österreichische rhetorische Sprachtraditionen wirksam (Friedrich Sengle), ${ }^{27}$ bis hin zu den weit ausgreifenden "Prunkreden", die sich von Sprachskepsis, Wortkargheit bis zum Verstummen abheben (so Herbert Seidler). ${ }^{28}$ Die jüngere Forschung achtet inzwischen stärker auf die verschiedenen

21. Gerhard Scheit, Grillparzer. Reinbek bei Hamburg 1989 (=rowohlts monographien 398), S. 7.

22. Ferdinand Kürnberger, "Grillparzer's Lebensmaske (23. 1. 1872)." In: Literarische Herzenssachen. Wien 1877, S. 297f.

23. Gerettete Ordnung. Grillparzers Dramen. Hrsg. von Bernhard Budde u. Ulrich Schmidt. Frankfurt/M., Bern - New York - Paris 1987, S. 18.

24. Gerhart Baumann, Franz Grillparzer (vgl. Anm. 16), S. 27.

25. Friedrich Gundolf, Grillparzer (vgl. Anm. 7) S. 9, 45, 68.

26. Joachim Kaiser, Grillparzers dramatischer Stil. München 1961.

27. Friedrich Sengle, "Franz Grillparzer." In: Biedermeierzeit. Bd. 3. Stuttgart 1980, Unterkapital: "Keine Sprachkrise im modernen Sinn", S. 115-118. 
Sprachebenen in Grillparzers Werk, die stilisierte und zugleich differenzierte Dramensprache; die, gemessen an der goethezeitlichen Lyrik, reflexionsbestimmte, zum Epigrammatischen neigende Sprache der Gedichte und die Sprache des Tagebuchschreibers, der kritisch scharf bis skeptisch beobachtet und analysiert. ${ }^{29}$ Im Bereich der dramatischen Form widersprach der These von der Aufweichung geschlossener Strukturen ("unaufhörlicher Beginn - unschlüssiges Ende") und einer sich darin abzeichnenden Modernität im Zeichen der Annäherung an die offene Form des Dramas (Baumann ${ }^{30}$ die These von der ausgeprägten Rahmenform Grillparzers (Seidler, zustimmend auch Sengle), ${ }^{31}$ die Traditionen der Geschlossenheit des Welttheaters weiterführt (Naumann). ${ }^{32}$

Hat sich hier das Miteinander von Widersprüchen eher im nachhinein aus dem Gegeneinander widerstreitender Positionen ergeben, so wird es z.B. bei Heinz Politzer als prägende Struktur Grillparzers anvisiert, wenn er im Bruderzwist das Miteinander von traditionsgestützter "Staatstragödie" und "moderner", der Psychoanalyse sich anbietender "Privattragödie" herausgearbeitet, so etwa im Verhältnis zwischen Rudolf II. und seinem Sohn Don Caesar im Bruderzwist ${ }^{33}$ : "die vermeintliche Geschlossenheit der Werke [Grillparzers W.W.] aufzubrechen, ihre Widersprüche nicht zu verbergen, sondern offenzulegen" (S. 18) wird zum Hauptanliegen "der historisch-kritischen Analysen" eines Sammelbandes aus dem Jahr 1987, dessen Titel bereits die Problematisierung eines früher (z.B. noch bei Sengle 1980) oft als "Qualitätsmerkmal" hervorgehobenen Schlüsselvorgangs in Grillparzers Werken enthält: "Gerettete Ordnung". Die Eröffnungssätze der Einleitung legen das programmatisch offen:

28. Herbert Seidler, Prunkreden in Grillparzers Dramen. Wien 1964 (= österreichische Akademie der Wissenschaften, Phil.-hist. Klasse, Sitzungsberichte, 244. Bd., 4. Abh.).

29. So bei Friedrich Sengle. Neuerdings auch bei Arno Dusini, Die Ordnung des Lebens. Zu Franz Grillparzers "Selbstbiographie". Diss. Wien 1989; gedruckt Tübingen 1991.

30. Gerhart Baumann, Franz Grillparzer (vgl. Anm. 16), bes. "Der dramatische Ablauf", S. 70-98.

31. Herbert Seidler, "Die Kunst der Rahmung in Grillparzers Dramen." In: Studien zu Grillparzer und Stifter. Wien - Köln - Graz 1970, S. 118-134; "Die Schlüsse in den Dramen Franz Grillparzers"; ebd. S. 135-150. Zustimmend zu Seidler auch Friedrich Sengle (vgl. Anm. 27), S. 68-71 ("Dramaturgie: nur kleine Zugeständnisse an die offene Form"), S. 112-118 ("Gibt es eine Formel für Grillparzers Dramatik? Keine Sprachkrise im modernen Sinn").

32. Walter Naumann, Grillparzer. Das dichterische Werk. Stuttgart 1956.

33. Heinz Politzer, "Grillparzers Bruderzwist. Ein Vater-Sohn-Konflikt in Habsburg." In: Festschrift für Bernhard Blume. Göttingen 1967, S. 173-194. 
Am Ende erscheint die Ordnung als gerettet, der in Konflikt mit ihr geratene Einzelne als zu Recht gescheitert oder wieder integriert: dieses Konstruktionsmerkmal ist Signum der meisten Dramen Grillparzers. Daher muß nach den Spuren ästhetischer Qualität nicht in der oft erzwungenen Schlußapotheose, sondern in den Bruchstellen und Widerständen der Stücke gesucht werden. Wie mühsam die aufgebrochenen gesellschaftlichen Gegensätze in der Restaurationsperiode literarisch verdeckt werden konnten, davon legen die Werke beredtes Zeugnis ab [...] [es] scheint von der neuerlichen Legitimation des alten Systems - durch Besserung oder Beseitigung des Störfaktors - nur wenige Ausnahmen zu geben. ${ }^{34}$

Als solche Ausnahmen werden dann herangezogen Des Meeres und der Liebe Wellen, worin das vorherrschende "Schema eindeutig um-" gekehrt wird: "Nicht das Individuum verschuldet seinen Untergang, sondern eine unerbittliche Hierarchie zerstört den ihr ausgelieferten Menschen"; weiter Weh dem, der lügt!, wo ein "Nicht-Gesellschaftsfähiger" vorgeführt wird, "der sich gegenüber den Mächtigen mit List behauptet". Und schließlich "das Trauerspiel Die Jüdin von Toledo, in dem den Großen vorgehalten wird, daß sie "Zum Versöhnungsfest I ein Opfer sich geschlachtet aus den Kleinen" (V. 1923f.).

Vergleicht man damit die Perspektive der zurecht gepriesenen Inszenierung der Jüdin von Toledo durch Thomas Langhoff bei den Salzburger Festspielen 1990, so wundert man sich über die im begleitenden Interview zur Fernsehaufzeichnung (gesendet am 5.1.1991 in FS 2) unterstellte Unvereinbarkeit zwischen dieser Theaterdeutung und der verächtlich abgetanen Interpretiererei der "Germanisten und Dissertanten", wüßte man nicht um den Topos der Germanistenbeschimpfung. Die Ambivalenz der herrschenden Ordnung, die ich bereits 1975 und erneut 1981 unter dem Titel "Thematisierung der 'Ordnung' in der österreichischen Literatur" von Grillparzer bis zur Gegenwart [bis zu Bernhard und Handke] behandelt habe, ${ }^{35}$ wird nun $\mathrm{zu}$ einem Leitthema der jüngsten Grillparzerforschung und bringt wirklich eine schwerwiegende Veränderung im Bild des österreichischen Klassikers mit sich.

Daran hat nicht zuletzt der Herausgeber der kommentierten Frankfurter Werkausgabe im Deutschen Klassiker-Verlag, Helmut Bachmaier, maßgeblichen Anteil, z.B. mit seinem Beitrag "Grillparzer: Ordo und Geschichte" (1988, 1991). ${ }^{36}$ Er deutet und bestimmt damit zugleich die "Klassizität" Grillparzers

34. Gerettete Ordnung. Grillparzers Dramen (vgl. Anm. 23), S. 9.

35. Walter Weiss, "Thematisierung der "Ordnung" in der österreichischen Literatur". In: Dauer im Wandel. Aspekte österreichischer Kulturentwicklung. Wien Freiburg - Basel 1975, S. 19-44; "Noch einmal "Ordnung" in der österreichischen Literatur". In: German Studies in India "Indo-German" 5 (1981), S. 16-31.

36. Helmut Bachmaier, "Grillparzer: Ordo und Geschichte (1988)". In: Franz Grillparzer. Hrsg. von Helmut Bachmaier. Frankfurt/M. 1991 (=st 2078), S. 259-270. 
historisch.

Grillparzers Dichtung besteht gewissermaßen aus zwei Primärtexten: aus dem 'klassischen' Text der Ordometaphysik und der Seinsherrlichkeit sowie aus dem 'modernen' Text der historischen Depotenzierung des Traditionalen und der Epochenanalyse des 19. Jahrhunderts." Seine "restaurative Klassizität" sei "letztlich zu beurteilen als der Versuch, Kunst und Kulturformen vor dem Andrang dekompositorischer Kräfte zu retten." "Der Begriff des 'Klassischen' gewinnt also seine wahre Bedeutung für Grillparzer aus der Abwehrhaltung gegenüber der geschichtlichen Lage, [nämlich der ästhetischen Kampfansage gegen den modernen Partikularismus] (S. 267f.)

6. Herrschende - Opfer, Große - Kleine, Einheimische - Fremde, Männer -Frauen

Einer solchen energischen historischen Festlegung Grillparzers und seines Werkes entspricht und widerspricht - zumindest scheinbar - die bereits erwähnte Inszenierung der Jüdin von Toledo durch Thomas Langhoff, der darin, ohne Gewaltsamkeit, durchaus aktualisierbare Widersprüche zwischen Herrschenden und Opfern, zwischen Einheimischen (Spaniern) und ausgegrenzten Fremden (Juden), zwischen Männergesellschaft und geopferten Frauen ausfindig macht und eine Parteinahme Grillparzers für die Opfer, die Fremden, die Frauen herausliest.

Ich halte diese Lesart für legitim und durch Grillparzers Werk über die Jüdin von Toledo hinaus vielfach und vielfältig gedeckt. Man hat Grillparzer immer wieder einseitig an Herrscher, Herrschen, Herrschaft ${ }^{37}$ an die Habsburger herangerückt, wenn man auch seine zunehmende Problematisierung der Herrschergestalten erkannte.

$\mathrm{Da}$ er die Gegenseite auch sah und thematisierte, daß zum "ganzen Grillparzer" auch hier der Widerspruch gehört, wurde demgegenüber zu wenig beachtet.

Über die bereits erwähnten drei Beispiele hinaus ist im Goldenen Vlie $\beta$ Medea, die Fremde, die Frau, das Opfer der herrschenden Kultur und der Staatsmacht, auch dann noch, wenn sie darüber zur Täterin, zur Mörderin wird.

Die Konstellation ähnelt der Jüdin von Toledo. Und hier wie dort gibt es auf der Gegenseite die Frau, die eins ist mit dem herrschenden System, hier Kreusa, dort Königin Eleonore.

Aber nicht nur Frauen sind die Opfer, denkt man nur an den "armen Spielmann" im Verhältnis zu seinem Vater, dem Vertreter der herrschenden Ordnung. Komplexer noch ist die Einheit von Herrscher und Opfer, die uns in Sappho, in Libussa begegnet, und in Kaiser Rudolf II., dessen Verwandtschaft mit dem "armen Spielmann" längst erkannt ist; bis zu einem gewissen Grade gehört

37. Zdenko Skreb, Grillparzer. Kronberg/Ts. 1976, S. 233. 
auch Ottokar hierher. Mit der Oppositionskonstellation von Herrscher und Opfer, Großen und Kleinen, Männern und Frauen, Einheimischen und Fremden verbindet sich eng eine regionale Konstellation, mit der Grillparzer in anderer Weise, als Joseph Roth es im Auge hatte, aber doch in seinem Sinn, die von Joseph II. geschaffene "moralische und geistige Basis für den späteren nationalistischen Hochmut der deutschen Österreicher gegenüber den anderen Österreichern" problematisierte. ${ }^{38}$ Kommt bei ihm doch das Herrschaftszentrum Wien nur wenig und kaum als solches ins Spiel (Der arme Spielmann), sondern eher aus der Perspektive der Kleinen und der Opfer, dagegen sehr wohl Böhmen, Ungarn, Spanien und - analog dazu - neben dem auf sich selbst bezogenen Griechenland das fremde Kolchis.

\section{Ausblick}

Die jüngsten Veränderungen des Grillparzer-Bildes haben den "österreichischen Klassiker" nicht gestürzt, sondern bestätigt, aber sie haben den Inhalt dieses Begriffs gründlich verändert. An die Stelle der Fixierung auf Tradition und Überzeitlichkeit oder eine ebenso einseitigen "Modernisierung" tritt die Kombination von historischer Situierung und immer wieder möglicher Aktualisierung. ${ }^{39}$ An die Stelle seiner Identifizierung mit dem bis heute wirksamen Modell der hierarchischen Ordnung des "größeren Österreich" tritt ein spannungsvolles Miteinander und Gegeneinander von Festhalten, Problematisieren, Aufbrechen und Überschreiten. ${ }^{40}$

Manches von dem, was wir im Zusammenhang mit der Entfaltung der Oppositionen von Herrschenden und Opfern, Großen und Kleinen, Einheimischen und Fremden, Männern und Frauen im Werk Grillparzers überlegt haben, läßt eine Verbindung Grillparzers nicht nur mit dem alten Humanismus, sondern auch mit einer Menschlichkeit $\mathrm{zu}^{41}$, deren utopisches Potential wir nicht übersehen und übergehen sollten.

38. Joseph Roth, "Grillparzer. Ein Porträt." In: Joseph Roth. Werke. Bd. 4. Köln o. J., S. 306-315, zitierte Stelle S. 309.

39. Dagmar Lorenz, "Grillparzers Lyrik im Kontext." In: Jahrbuch der GörresGesellschaft 16 (1984-86), S. 37-56; Hans Höller, "Der "Todesarten-Zyklus" des 19. Jahrhunderts. Ingeborg Bachmann und Franz Grillparzer." In: Jahrbuch der Grillparzer-Gesellschaft 15 (1983), S. 141-153; Weiter die Inszenierung der Jüdin von Toledo durch Thomas Langhoff.

40. Helmut Bachmaiers Begriff: Grenze und Grenzüberschreitung. Sämtliche Werke. Text und Kommentar. Hrsg. von H. Bachmaier. Bd. 2. Frankfurt/M. 1987, S. 618-634.

41. Vgl. dazu Zdenko Skreb, Grillparzer (vgl. Anm. 37), S. $235 \mathrm{f}$. 